\title{
Evaluating the Credibility of Arabic News in Social Media through the use of Advanced Classifier Algorithms
}

\author{
Mai A. Fadel \\ Computer Science Department, Faculty of Computing and Information Technology, \\ King Abdulaziz University, Jeddah, Saudi Arabia \\ mfadel@kau.edu.sa
}

\begin{abstract}
The aim of this paper is to evaluate the credibility of Arabic Social Media News based on classifier models and integration tools for automatic feature extraction such as image recognition and natural language processing for comments in order to provide an initiation of a large dataset. The proposed methodology involves building labeled data set consisting of Arabic news in twitter, gathering features from previous studies, extracting features, conducting a number of experiments to choose the classifier algorithm and features set to reach the highest accuracy and finally build and test the purposed system. Based on the selected classifier model, the system processes the tweet's URL and classifies it into credible or non-credible news.
\end{abstract}

Keywords:Social media, feature extraction, classifier model.

\section{INTRODUCTION}

The Internet has revolutionized people's lives. Statistics showed that 4.01 billion of the world population use the Internet (1)while 181 million users in Arab countries (2)and 26 million out of 32.28 million use internet in Saudi Arabia (3). These days many people have turned to websites and social media whose content is integrated into search engine results to check the news and they regard it as a reliable source of news, and it is difficult for them to distinguish between the credible news from the rumors since the Internet is a free access to everybody and they can share news and information regardless of their experience and without scrutiny. So, it is important to distinguish rumors to reduce their separation which can cause negative consequences, for example spreading the fake news about crises may cause stir among people while the credible news may lead to save lives in disaster times.

Furthermore, social media has become an integral part of our daily life. News and interactions with others via social media affect a considerable proportion of our emotions and decisions. Such news and information are communicated by authorities and individuals as well. Due to the sensitivity of the effect of such news, and varied spectrum of anonymity of the source of news, there is a need for means to evaluate the credibility of news in social media. Also, there are limited researches on credibility assessment of news in Arabic world.

Our solution is based on the available information related to the news (or post) that called features either related to its content, its author or any external sources that deny or prove the news to evaluate the credibility of the news. By using Machine Learning concepts to analyze these features to classify the news using classification model, also taking advantage of the huge amount of news and data on the internet that is useful in training the model. Therefore, we propose building a tool that helps in classifying the Arabic news in Twitter to credible or non-credible.

The objectives that will be followed to achieve the main purpose of the paper are the following:

(i) Identify the features: define a systematic way to gather the features that effects the credibility of the news, (ii) Prototype modeling: build a simulator system by using an existing data set, (iii) Gathering data set: collect dataset to test the classifier, (iv) Features extraction: develop a mechanism to extract features from the dataset, (v) Features set determination: choose different sets of features to make different experiments, (vi) Classifier: choose the most appropriate classifier algorithm then build it and train it, (vii) Testing: evaluate the proposed system and (viii) Integration: build the integrated tool.

The paper is organized as follows: Section II presents the paper motivation and related state of the art. Section III presents more details about credibility features selection. Section IV presents the developed system prototype, while section $\mathrm{V}$ describes the reletaed validation experiments. Then, section VI discusses the data collection procedure and section VII presents the extraction mechanisms of the selected features. Section VIII then presents the conducted experiments using the developed model and section IX demonstrates the achieved results. Finally, section X concludes the paper.

\section{RELATED STATE OF THE ART}

The subject of verifying the credibility of the news has of great interest since ancient times, which is one of the applications that using the machine learning and classification algorithms. It started with journalists with different ways to verify the credibility of the news and their roles in gathering information from the source. Then it 
started to appear in other types of media sources, such as newspapers, TVs, websites and social media like twitter, Facebook, Instagram...etc. The news credibility of the Internet content is becoming very important. Recently, many researches have analyzed media credibility scales for news and the most influential factors in the credibility of news for different types of media: newspaper, televisions and online credibility. Generally, they agreed that credibility is a component of five: believability, accuracy, trustworthiness, unbiased, completeness, and their impact difference from one type of media to another (4). This section will present the background of credibility assessment concept, starting with the definition of credibility, credibility features, assessment model and data.

The tweet is a message that has been posted in twitter, with a limited length up to 280 characters. Tweets can include text, images or videos and other information about the tweet, such as number of retweets and number of likes. User side includes user information such as name, location, profile image and account verification.

To clarify the meaning of credibility, first the social media must be defined as the web services that allow users to construct a profile and share contents and connection with other users. These connections and type of content may vary from site to site such as Twitter, Facebook etc. (5). (6)had defined two types of "credibility" in twitter: (i) Tweet-Level Credibility: a degree of believability that can be assigned to a tweet about a target topic, i.e.: an indication that the tweet contains believable information and (ii) Social Credibility: the expected believability imparted on a user as a result of their standing in the social network, based on any and all available metadata.

Features are one of the most important influential factors in the topic of evaluating the news credibility, this section will present the most common credibility features in the previous studies with their impacts on the assessment. Finally features extraction mechanisms are explained.

The credibility features are the features set that are related to the news to be evaluated, whether it is a tweet, an image in Instagram or an article on a website. Some researchers care about selecting the minimum number of features while others care about collecting the most important features that impact the assessment regardless of the number. Most researchers agree to divide the features into two types: (i) content features such as, ones with URL, ones that have informal words...etc, and (ii) author features which are related to the author/ source of the content, such as profile images, names, and comments which are powerful feedback to evaluate the experiences and opinions of the author (7)(6). There is a third type mentioned in some studies is the user features which is about user background and preferences.

According to (7), the 45 common features proposed for Twitter, features of content are: topic of content, the date, number of favorite, number of hashtags, number of retweets, spelling errors, number of question marks, number of emoticons, number of exclamation marks, number of characters, number of words, number of special characters, includes URL, includes image, number of pronounces, starts with a question, includes laughing words, number of dialects words, number of bad or swear words, number of religious words, number of swear words, includes dialects or bad or swear or religious words, number of formal words and includes urgent words. For author features are: verified account, using default image, following number, followers number, following to followers ratio, tweets number, favorites number, tweets to favorites ratio, has a bio, has a name, has educational information, has a position of employment, bio has contact information, bio has education or work or contact information or at least two of them, has webpage, year of joining, name, bio and location related to the topic, and bio has special characters. Then by applying different kind of features algorithm, the number of features became less.

Other researchers suggested less number of features such as (8)who proposes 5 features: the similarity with verified source, excluding inappropriate words and provides link to verified sources for content features, and for author features such as: verified account and degree from TwitterGrade.com. Moreover (9)proposed 13 features, (10)proposed 13 features for content and author, and (11) proposed 20 features for content, author, topic, and diffusion.

The impact of feature in evaluating the news credibility is the main factor that affects choosing it as a credibility feature. Most of the researchers select the features based on surveys such as (12), experiments by number of participants, or most common features from previous researches such as (7).

Some researchers first select the set of features then, selecting subset of features from the previous features set based on different approaches by using: a survey results, or an algorithm for selecting features, such as the best first search, chi square algorithm, Relief algorithm and Correlation attribute as proposed by (7). Other studies depend on the final result for each features and set of features to know the most effective features that lead to higher results, such as: (13)who implemented the classifier on different feature set each time to show the effectiveness of feature sets, as feature of content resulted high precisions but also resulted to low recall value. Also (8) show that the average recall value appears higher in some features compared to small effect of the other features. Extract features from the dataset can be internal, which is related to the content, or external which is unrelated to the content, such as author features and user features. Internal features are mostly related to linguistic features and textual analysis that needs to perform a text preprocessing by stemming 
word, normalizing, removing stop words, or part of speech ...etc. as will be explained in data preprocessing. The other features such as: hashtags which can be extracted in twitter by using API, verifiability feature of the content which is calculated by similarity with a trusted source by using Cosine or Jaccard similarity measures (8)(10)and for containing URLs feature first unshorten the URL by using specialized sites then checking by experts or by using Web of Trust (WOT), a website that checks other websites' reputation ratings (14).

External features such as author features are easier to extract by API, Twitter's API downloads text messages and metadata, such as date of tweet, number of retweets and information about the author (e.g. name, number of followers, number of following and bio information) (14).

As explained in one of the previous research on the features extraction process (8), has created a matrix to store features after extraction in the database to feed into the classifier. After preprocessing, the text is stored in vector of words to implement it in the classifier to be able to deal with. One of the most common approach to transfer the text to vector of words is Bag of Words (BOW) file which counts the number of times that word appears in the document. By using Termfrequency-inverse document frequency TF-IDF which replaces the word counts by scores towards the whole dataset (15). Researchers have used classifying models (16), (6) and ranking models (17), (14), (10). A ranker's main objective is to sort data or objects based on some criteria, however, it can be used to solve other problems like classifying. Some researcher has used ranker such as SVM ranker, AdaRank, RankBoost, and Classifiers such as SVM, Decision tree, Naive Bayes, which are described in the following.

Classifiers are widely used in assessing the news credibility, mostly binary classifiers which classify the news to only credible or non-credible news. However, some researchers used multiclass classifier as in (16) who classified the news to four classes highly credible, highly non-credible, neutral or controversial.

Support vector machine (SVM), is a supervised classifier that plots the labeled data (training dataset) as points in a multidimensional space, then it constructs hyperplanes to separate the data to different classes. The new data (test dataset) is mapped to the space to predict to which class it belongs as shown in Figure 1. The optimal hyperplane is the one that separates the classes with the largest margin (18).

Naive Bayes (NB) Classifiers are set of probabilistic classification algorithms that are based on Bayes' Theorem which many modern machine learning techniques rely on Bayes' theorem, which is a formula that describes the probability of an event, based on prior knowledge of conditions or evidence (19). For example, assume that a hypothesis A and evidence B, Bayes' theorem describes the relationship between the probability of the hypothesis before getting the evidence and the probability of the hypothesis after getting the evidence.

$$
\text { Bayes' Theorem } \quad p\left(\frac{A}{B}\right)=\frac{P\left(\frac{B}{A}\right) P(A)}{P(B)}
$$

In all the NB classifiers, there are two fundamental assumptions

- the features are independent, in other words it assumes the existence of one feature if it is distinct from the existence of any other feature.

- each feature contributes as much as the other features to the outcome.

NB is a simple model easy to build, fast in prediction, useful for large datasets and for multiclass prediction (18).

Iterative Dichotomiser 3 Decision tree (ID3) is an algorithm used to generate decision tree, the tree classifies the data based on some features or attributes. The decision tree consists of two types of nodes decision nodes and leaf nodes. Each decision node tests a feature then splits it into more than one branch which could be another decision tree or leaf nodes, where the leaf nodes are the classes. To decide which feature goes into decision node the ID3 calculate the information gain or the entropy for each feature and choose the one with the largest information gain.

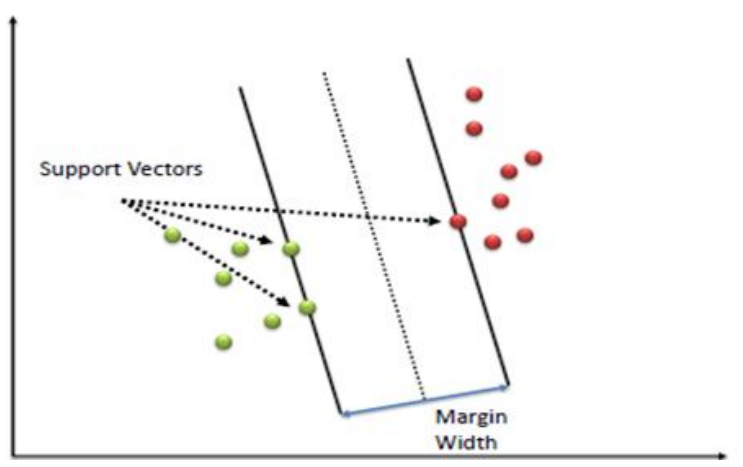

Figure 1: SVM Classifier

Building and testing the classifier and system will need data that is labeled to credible or non-credible as an input to our system.

Social media companies like Twitter, Facebook, Instagram, Google...etc., have created Application Programming Interface (APIs) with big capabilities and huge numbers of queries to extract data. These APIs are free, accessible to anyone, help developers to display content in their apps, and help researchers collect data. There are also some open source crawlers and tools that help to collect the data from the APIs such (15)who used Hoaxy which use the Twitter API to visualizes the spread of articles online.

Most of the news credibility researchers have collected the data from these APIs especially from Twitter API or using tools as mentioned above (14). Other researchers collected 
data on specific topics or keywords or on trend topics. Others collected data focusing on certain type of users or publishers (6).

Few researchers collected the data manually, such as (12)they collected 18 tweets for 266 volunteers using twitter search. But this approach consumes time and provides small number of data.

Some researchers prefer to use datasets from databases like The Global Database of Events Language and Tone (GDELT), Kaggle and other databases, like (15)who collected more than 124000 article from GDELT, most of these datasets are in English language. Disadvantage of these databases is that it does not provide real time data like the APIs, also it does not allow the researcher to control the data as much as in the API. Some others build new data set using the APIs or manually. It is based on processing on raw input data to transform it into a format that is more effectively processed for user purpose, and it is considered as a data mining technique. It is an important step before building and training the classifier on the data. Data preprocessing depends on the type of data as image preprocessing and text preprocessing, but most of the researches focus on text preprocessing which is the common content in the Internet and social media.

Text preprocessing consists of many steps depending on the purpose of the researcher and it needs to use Natural Language Processing (NLP). Over domain news credibility researches use the following steps of text preprocessing:

(a) Removing stop word: commonly used words (such as " and, any, the...etc." and in Arabic such as"ذو، ثم، نحن wo..."). Most of the researchers use an existing list of stop words in the target language.

(b) Part-of-speech tagging: which determines grammatical category of the token for an ambiguous word according to their context (17)(20), and most of researches using an existing tool to as Stanford Part of Speech (POS) tagger for Arabic language which is tool written in Java to obtain the Arabic POS tagger (8).

(c) Stemming word: a stem word is the word form without any inflectional suffixes or affixes are added. Most of the researches using an existing tool developed based in stemming algorithms to obtain the stem words.

(d) Determining the content that has relevant information to purpose study and cut off the remaining.

(e) Normalization.

(f) Contraction expansion (such as "can't" becomes "cannot") (g) Punctuation removing: (15) removing apostrophes, brackets, colons, commas, dashes, ellipsis, exclamation marks, periods, question marks and other typography marks.

There are few studies that preprocess manually but often they are simple studies on a small data sample because it is hard work which needs experts and it take a long time to do. Therefore, many tools have been developed to do this work like the Java-based library named AraNLP that covers various Arabic text preprocessing tools which gather most of the Arabic text preprocessing tools into one library (21), and other helpful packages in English language such as scikitlearn, pymongo, and spacy.

Generally data annotation is known as labelling any type of data images, audio, text, video, ...etc., For example, if the purpose of the program is to identify cats in images, the system will have a large number of photos labeled as cat or not (22). The annotation is important in the supervised machine learning where the dataset used in training should be labeled so the machine can understand and then predict or classify new unlabeled data which is called the test dataset.

When the researcher uses an available dataset it will be annotated, the problem arises when gathering data manually or from API, then the researcher has to annotates the data. Most of the researches in the news credibility annotated the data manually with help of volunteers. There are some researchers who used systems or tools to do this task, such as (23) who used Amazon Mechanical Turk (MTurk) to annotate the tweets to chat or news and then annotate the news tweets to credible or non-credible, MTurk is marketplace which enables users to coordinate the use of human intelligence to perform tasks that computers are currently unable to do. These tools help save time, but they are mostly for the English text, they can be also helpful to label images.

There are many tools that can help build classifiers or provide built-in classifier, we will present the most common of them, and will compare them to determine the most appropriate one to our needs.

WEKA is a free and open source platform that provides collection of machine learning algorithms and supports them in java package, WEKA provides a graphical user interface and it allows to build classifiers or use one of the built-in classifiers. RapidMiner is a partially free platform that can be used in machine learning, deep learning and data mining ...etc., RapidMiner provides a graphical user interface and it allows to build classifiers or use one of the built-in classifiers. $\mathrm{R}$ language is a free and an open source programming language that can be used to statistical computing and data analysis, by using Rstudio as a development environment or $\mathrm{R}$ tools in visual studio. $\mathrm{R}$ language does not provide built-in classifiers nor graphical user interface. 
The work (23)proposed 68 features to determine the credibility of tweet and grouped them into four categories (message-based features, user-based features, topic-based features and propagation-based features), then they used best-first feature selection algorithm to choose the best fifteen features. The authors used data set collected from twitter to monitor over 2500 different topics, and mechanical Turk evaluators to determine if the tweet is newsworthy, if so indicate its credibility level. Then they trained two supervised classifiers. The first one to determine if the tweet is newsworthy, and the second one to predict the credibility level of the tweets. They tried number of algorithms including SVM, ID3 decision trees, decision rules, and Bayes networks. The highest accuracy achieved was $89 \%$ by ID3 decision for the first task, and $86 \%$ also by ID3 decision tree for the second task. Finally, they used the same classifier on different subset of features (text-subset, network-subset, propagation-subset, top-element-subset) to compare which subset has more impact on the credibility and it turned out to be the propagation-based features.

The authors in (6) presented and evaluated three different models, the first model focuses on credibility at the user level, the second model on credibility at the content level, and the last model combines facets from both models in a hybrid method. They collected data from Twitter streaming API, focusing on seven specific topic data sets, and manually annotated 5000 tweets. For the first model, they proposed six features and incorporated them in a weighting scheme. For the second model, they used nineteen features approximately half of them were taken from a 2011 study by Castillo et al. In the last model, they used four different hybrid methods to evaluate the credibility. Each strategy was represented as a set of weighted features and loaded as an input file to WEKA tool and ID3 decision tree learning algorithm was used. The user-based model outperformed the other models achieving an accuracy of $88.17 \%$.

The authors in (14) built an extension to chrome browser (24) to check the credibility of tweets and give a score between 1-7. They collected the data from twitter API using specific keywords for six prominent events and annotated 500 tweets using crowd-sourcing provider CrowdFlower. The features they choose can be driven from the tweet automatically since they aimed to build a real time system. In total there were 45 features and they used multiple learning-to-rank algorithms. The best was SVM-rank semisupervised which gave 0.72 NDCG (Normalized Discounted Cumulative Gain) in 9-10 seconds. By the time they published the paper, the extension was installed and used by 717 users in three weeks, TweetCred displayed $84 \%$ of the tweets credibility score in less than 6 second and the user's agreed with $43 \%$ of the tweets credibility score given by TweetCred.
The work (7) studied the credibility measure for microblogs in Arabic language. The dataset built from a large corpus of tweet messages gathered using NodeXL (Twitter Search API tool). 199 unique tweets in 9 different topics, participants labellers independently labeled them, by using forty-six features, 24 for content features and 22 author features. To classify the messages credibility, ID3 Decision Tree classifier algorithm was used within Weka tool and evaluated three types of datasets:

(i) Labeled dataset obtained using simple majority voting an accuracy rate of $(51.25 \%$ - 59.799) from its different classes

(ii) Dataset with labels values obtained after applying selective labellers' weighting measures, using Cosine algorithm which measures the similarity between two sets represented as vectors in multidimensional space by measuring the cosine of the angle between them, with an accuracy rate of $55.78 \%$ and Jaccard algorithm which measures the similarity by dividing the intersections between the sets by their union with an accuracy rate of $56.78 \%$, and using Standard Deviation accuracy which has an accuracy rate of 55.28\% and Variance accuracy which has an accuracy rate of $60.80 \%$ for accuracy measures.

(iii) Labelled dataset obtained with proposed labellers' weighting aggregation model where compared between accuracy by using 3 famous feature selection algorithms from Weka: Chi square (X2) which statistically measures the independence between the feature and the class (label), that results thirteen features with an accuracy rate of $63.31 \%$, Correlation Attribute which drops one of two features if they have high correlation because they have the same effect, that results nine features with an accuracy rate of $64.32 \%$, and Relief algorithm which calculate a score for each feature and excludes it if it is less than the threshold, that results fourteen features with an accuracy rate of $65.82 \%$.

The results show that the classification rate of the proposed model $(59 \%-66 \%)$ is more desirable than the classification rate of simple majority voting approach. Using Relief Algorithm is the most accuracy rate to select features.

Finally, (25)built a system (CAT) which automatically predict the credibility of a given Arabic tweet. To build the system they collected 9000 Arabic tweets and built their own crowd-sourcing to annotate the tweets. The feature set composed of 26 content-based features and 22 user-based features. They trained the classifier using multiple algorithms SVM, Naive Bayes, random forest, and performed 10-fold cross validation, the best was random forest with 75.8 WAF, the Weighted Average F-measure (WAF) is the sum of each F-measures weighted according to the number of instances with that particular class label, which the F-measure expresses the average of the both precision and recall (25)(26). They also trained the classifier using the user-based features only and got 68.9 WAF and using the content-based features and got $68.7 \mathrm{WAF}$. 
Mai A. Fadel, International Journal of Advanced Trends in Computer Science and Engineering, 9(4), July - August 2020 , 6088 - 6102

\section{CREDIBILITY FEATURES SELECTION}

This section will present the selected features set and the methodology to choose it, as well as the related extraction techniques for these feature sets. The features that have been chosen in order to implement their extraction mechanisms and to build the dataset, and to use them in the experimentation of different classifier algorithms to reach to the final set of features that has the highest accuracy.

Table 1: Features Set

\begin{tabular}{|c|l|}
\hline Feature & Type \\
\hline 1-Verified account & author \\
\hline 2- image user is default & author \\
\hline 3- tweet has URL & content \\
\hline 4-tweet has an image & content \\
\hline 5-number of characters in tweet & content \\
\hline 6-num of followers of the author account & author \\
\hline 7- num of following of the author account & author \\
\hline 8- number of words in tweet & content \\
\hline 9-username of author & author \\
\hline 10-num of retweets & content \\
\hline 11-number of favorites & content \\
\hline 12- length/num of words of the tweet & content \\
\hline 13- Date of account creation & author \\
\hline 14- number of question mark in the tweet & content \\
\hline 15- tweet has positive words & content \\
\hline 16- tweet has negative words & content \\
\hline 17-tweet has bad/swear words & content \\
\hline 18-protected account & author \\
\hline 19- the tweet is replay (Quote tweet)? & content \\
\hline 20- author's number of tweets & author \\
\hline 21-author has web page in the bio & author \\
\hline 22- author's bio has location & author \\
\hline 23- author list number & author \\
\hline 24- number of Hashtags in the Tweet & content \\
\hline 25-number of exclusive marks in the tweet & content \\
\hline 26- author's number of favorite tweets & author \\
\hline 27- Related image to tweet content & content \\
\hline 28- Comments' polarity & content \\
\hline 29- following to followers ratio & author \\
\hline 30- URL source is related to tweet content & content \\
\hline
\end{tabular}

First we collected the most common features from previous studies in the literature review, as suggested by these researches(6),(16), (7), (14), (12), (8), (10), (23), (27) and (11). The records $1-26$ in Table 1 show the most common features that have been selected.

In addition, there have been other promising features that had good impact on the accuracy from one of the recent previous studies (10) which is the 'Users' Comments Polarity" meaning that the tweet's comments can be calculated with the help of sentiment analysis approach to know if they agree with the content of the tweet or not which has a high effect on credibility assessment. The length of screen name feature has been modified to the extent of similarity between screen name and username of the author (Author name similarity feature).

Also, some other features have been added based on another feature that will be more effective in assessment of the credibility such as: Related Image feature which most of the researches suggested "Tweet has an image" feature without checking the content of the image and its relationship to the tweet. The records 27-30 in Table 1 show the special features that have been selected.

The features extraction mechanisms were implemented in order to automatically extract the features of the gathered data to provide the large dataset in an easier way.

The features set extraction are divided into two types: 
Mai A. Fadel, International Journal of Advanced Trends in Computer Science and Engineering, 9(4), July - August 2020,6088 - 6102

(i) Features that got directly with the tweet from the twitter's API

(ii) Features that need to implement an algorithm to extract it

\section{SYSTEMPROTOTYPE}

The developed system receives the URL of the tweet as input and extracts its features and then uses the trained classifier to predict the tweet credibility as shown in the flowchart (Figure 2).

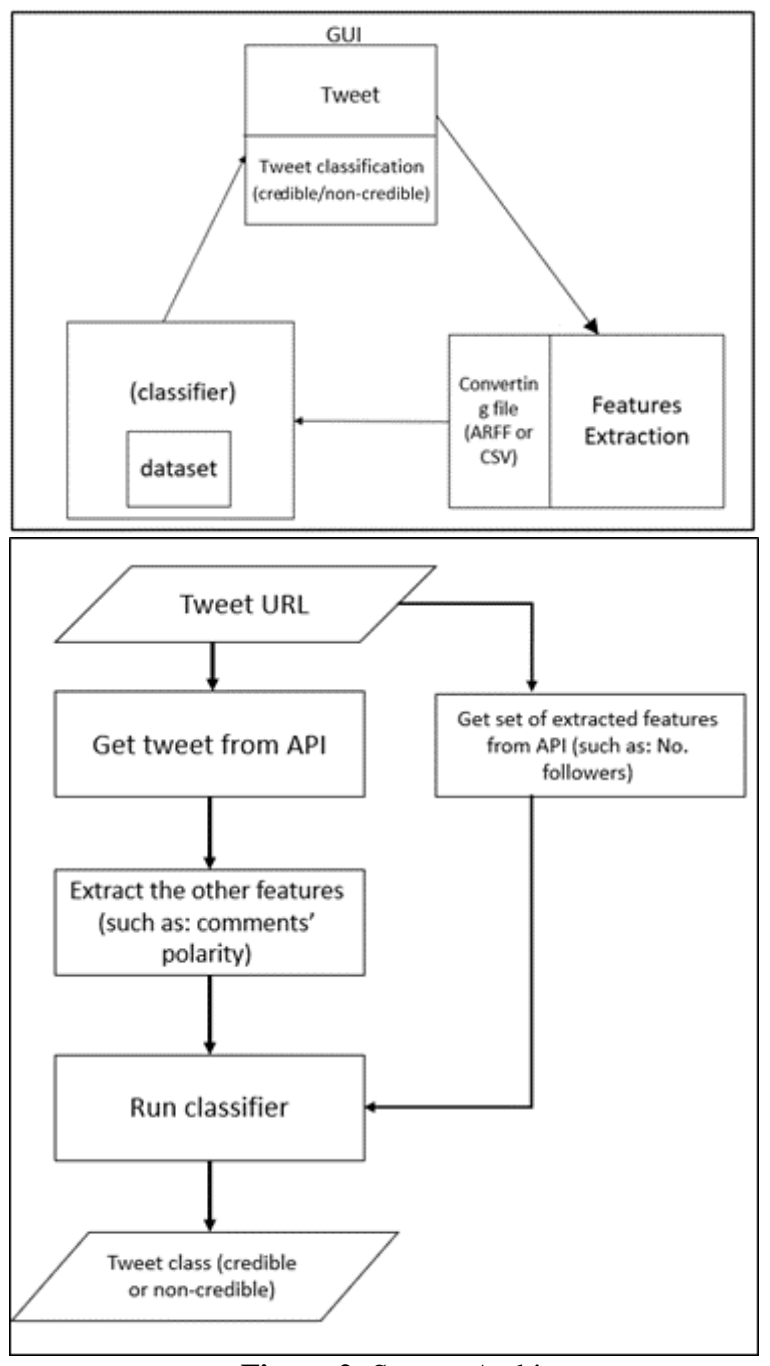

Figure 2: System Architecture

The System architecture (Figure 2) consists of three modules, the first module is the GUI which receive the URL from the user and send it to the second module which extract the features from the tweet then send it to the third module as a file. The third module is a trained classifier which will classify the tweet and return the class to the GUI module.

The GUI component (Figure 3) is related to the credibility assessment. It only shows the class of the tweet while the assessment model (classifier component) is hidden.
In order to build the system, first a dataset was obtained to be used by the classifier in this phase, then the classifier model was built using several algorithms and, finally the GUI was built.

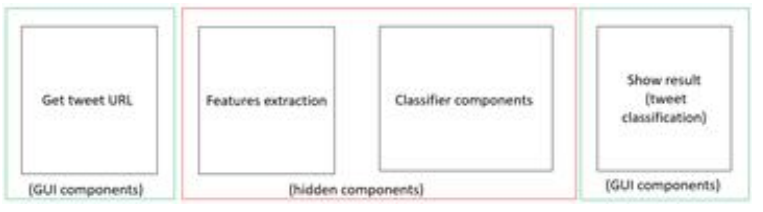

Figure 3: The proposed System GUI Component

The dataset used in this phase was obtained from (28). It contains tweet in English and Spanish language, which will be replaced in the future work with Arabic tweets. The data waslabeled as real, fake, or humor and consists of approximately 15000 records, and consists of multiple files with different attributes. The needed attributes. The following attributes are necessary:

1- $\quad$ Tweet id: the ID number of the tweet as given by twitter

2- num_words: number of the word in the tweet including hashtags and mentions but excluding links.

3- text_length: number of the characters in the tweet

4- contains_questmark: whether the tweet contain question mark '?' or not

5- $\quad$ num_questmark: number of the question mark '?' in the tweet

6- contains_exclammark: whether the tweet contain exclamation mark '!' or not

7- num_exclammark: number of the exclamation mark '!' in the tweet

8- contains_happyemo: whether the tweet contain any happy emotions

9- contains_sademo: whether the tweet contain any sad emotions

10- contains_firstorderpron: whether the tweet contain the first order pronoun

11- contains_secondorderpron: whether the tweet contain the second order pronoun

12- contains_thirdorderpron: whether the tweet contain the third order pronoun

13- num_uppercasechars: number of the uppercase characters in the tweet excluding 'RT' acronym

14- num_possentiwords: number of the positive sentiment words in the tweet

15- num_negsentiwords: number of the negative sentiment words in the tweet

16-_num_mentions: number of mentions (@user) in the tweet

17- num_hashtags: number of hashtags (\#hashtag) in the tweet

18- num_URLs: number of the URLs in the tweet

19- num_retweets: number of retweets to this tweet

20- tweet_id: the ID number of the tweet as given by twitter 
21- num_friends: number of following (users followed by this user)

22- num_followers: number of followers the user has

23- folfriend_ratio: the ratio of the following/followers

24- times_listed: the number that the user was listed in

twitter

25- has_url: whether the user has URL in his profile or

not

26- is_verified: whether the user is verified by twitter

27- num_tweets: the number of tweets the user has posted

28- Label: only three possible values (real, fake, humor)

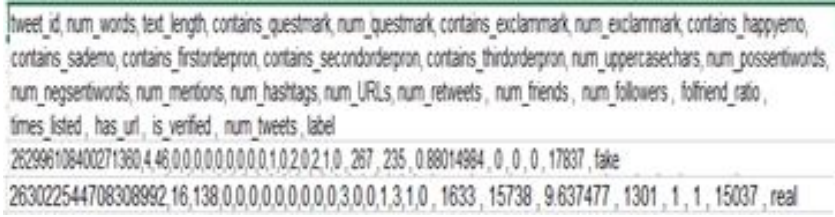

Figure 4: Sample of the Dataset

The interface snapshots are shown in Figure 5.

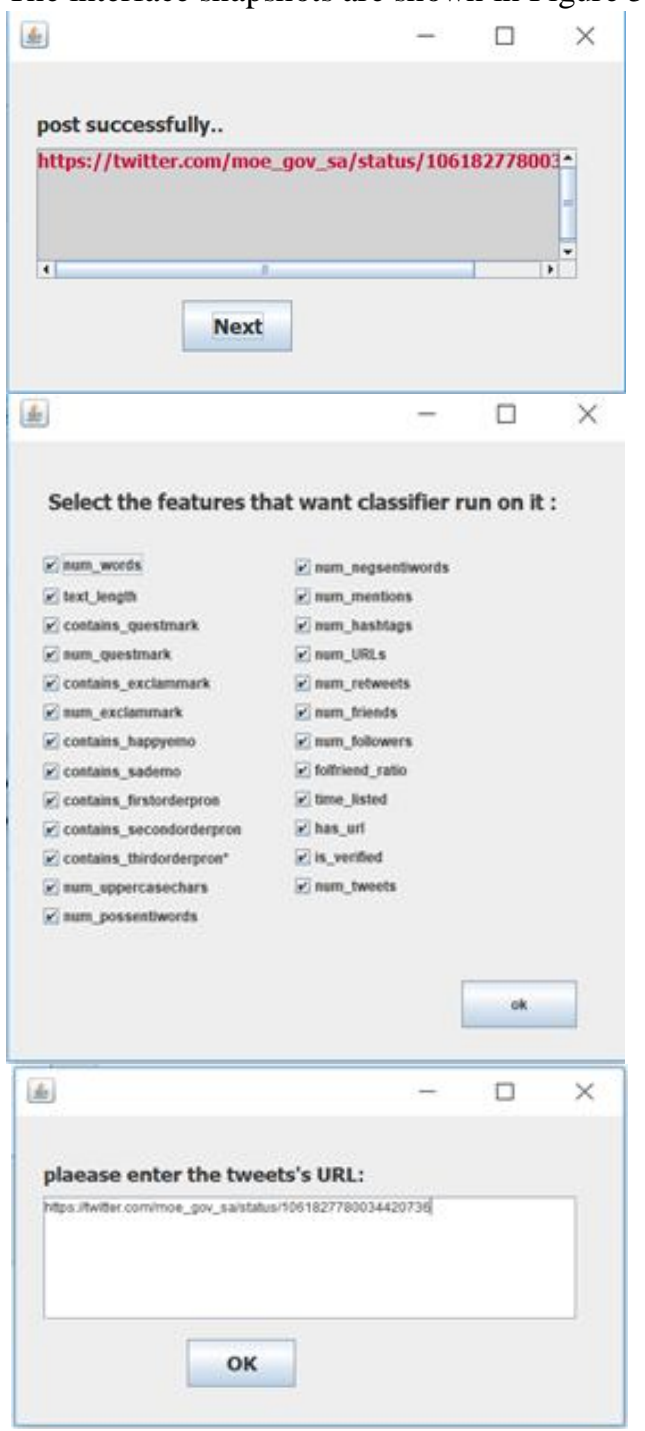

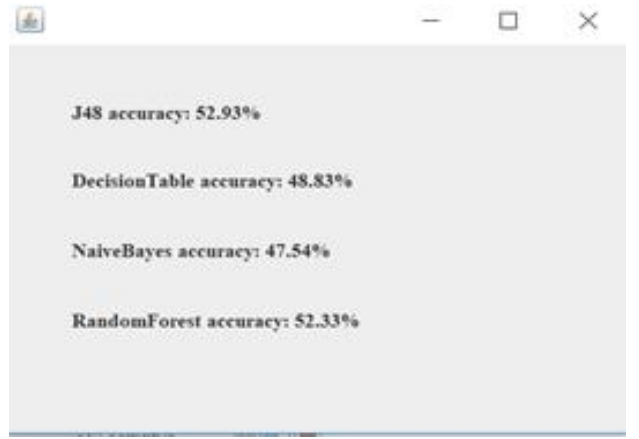

Figure 5: User Interface snapshots

\section{DESCRIPTION OF VALIDATION EXPERIMENTS}

The classifier algorithms used to conduct the experiment are Naïve Bayes, Decision Table, Random Forest, and Decision Tree ID3 which have been used in many researches such as in (23), and (16).

By using the built-in NaïveBayes, DecisionTable, RandomForest, and J48 (Decision Tree ID3) form WEKA and performing 10-fold cross validation strategy to conduct 3 different experiments:

(i) Experiment 1: Run the classifiers on all the features in the obtained dataset. The following results were obtained in terms of Algorithm Accuracy:

Naïve Bayes $\quad 48.36 \%$

Decision Table $52.58 \%$

Random Forest $54.60 \%$

Decision Tree ID3 $53.66 \%$

(ii) Experiment 2: Run the classifiers on content features only which are18 features. The following results were obtained in terms of Algorithm Accuracy:

Naïve Bayes $\quad 45.69 \%$

Decision Table $52.20 \%$

Random Forest $52.82 \%$

Decision Tree ID3 $\quad 52.70 \%$

(iii) Experiment 3: Run the classifiers on author features only which are 7 features. The following results were obtained in terms of Algorithm Accuracy:

Naïve Bayes $\quad 50.12 \%$

Decision Table $49.08 \%$

Random Forest $49.39 \%$

Decision Tree ID3 $\quad 45.90 \%$

Based on the abovementioned results, Experiment \#1 has the best accuracy whereas in Experiment \#2 and \#3 the number of author features is very small compared to number of content features, for that reason they have lower accuracy.

\section{DATA COLLECTION}

This section presents the data collection phase in order to build the proposed system and describes how data is collected, annotated in order to build the dataset after extracting the features from it, and then use the dataset to train the classifier model. The data of the system are tweets 
from Twitter and should be cover the two type of news credible and non- credible to be assessment. There is no available Arabic news dataset so, the solution to collect and build Arabic dataset.

This section will discuss the data collection process step by step.

A twitter developer account must be created to get the 4 credentials keys: API key, API secret, Access token and Access token secret for accessing Twitter API. It took 2-3 days for accepting the request of the application. There are many libraries for accessing the Twitter's API, and the used tool is Tweepy is Python library.

By using an existing code to collect tweets from (29) and the code from (30) that retrieve comments' tweet and by integrating them together with some modifications to suit our purpose, a code was implemented to retrieve tweets depending on the user, language or specific words.

The output of this code are two CSV files which contain one for tweets jsons and another for the comments which they are connected by tweet's id.

The approaches that followed in the data collection are by retrieving tweets of specific user or tweets that contains specific words or phrase, because the way of getting stream of tweets from the timeline without any filtering will retrieve a huge number of random data that is not needed and useless which will consume a time to clean it.

The data was collected for two weeks approximately starting from 24 Jan 2019.

The total of collected data is 1162 tweets, 808 credible tweets and 354 non-credible tweets.

The collected data was in formal Modern Standard Arabic Language (MSA) to facilitate the text mining and analysis process.

The approach that followed to annotate the data is selflabeling based on some criteria that will be explained in the coming subsections. The tweet is considered as credible if the news of the tweet has been published or mentioned by a trusted source whether another account, news site or scientific paper. Figure 9 shows a credible tweet as an example.

The tweet is considered as non-credible if:

(i) The news denied by a trusted source. Figure 10 and Figure 11 show a rumor that got denied by Saudi food and drug authority.

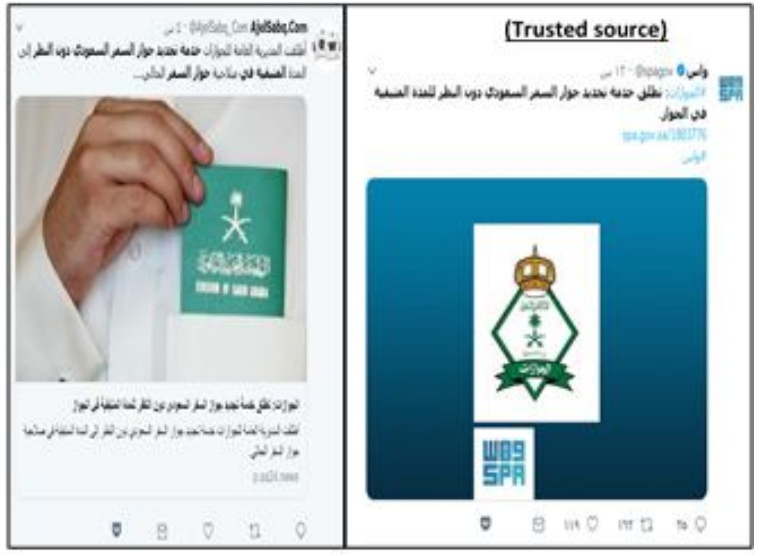

Figure 9: Credible Tweet Example

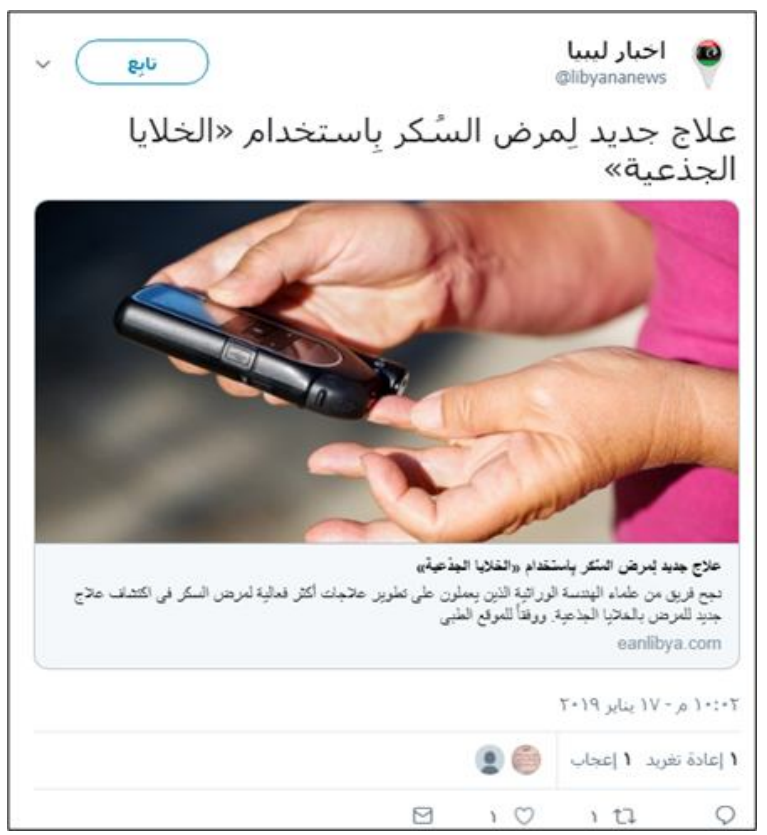

Figure 10: Rumor Example

(ii) The news denied by accounts that are interested in denying rumors in social media (Figure 12). These accounts deny the rumor by getting the reel news or by proofing it as non-credible from another trusted source, as illustrated in Figure 13.

(iii) The news is spread as an official news although the official authority did not announce it. (For example, there was an aircraft carrying a football player named EmilianoSala disappeared, some accounts in twitter announced finding the player body freezing in the sea although the search team did not announce finding the body and continued to search). 


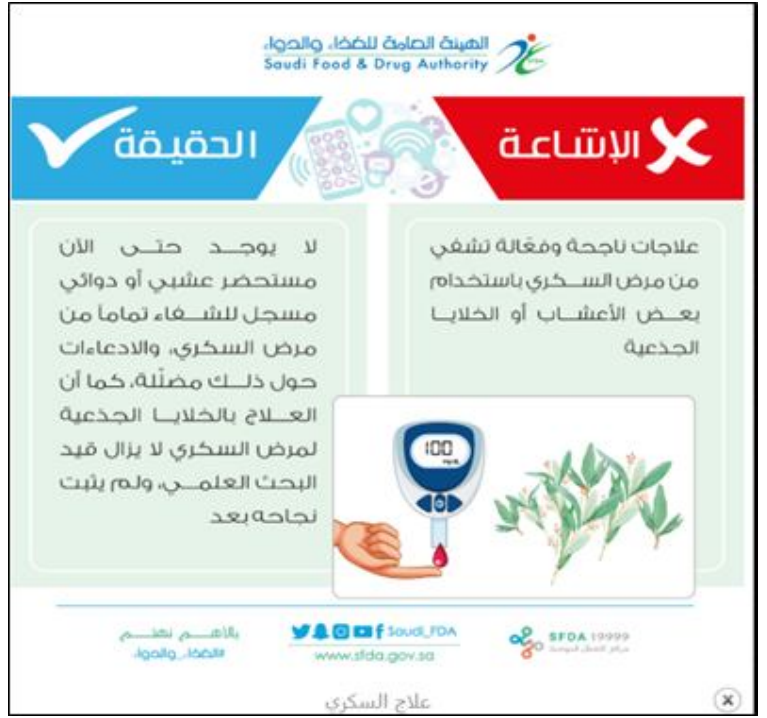

Figure 11: Saudi Food and Drug Authority Denial
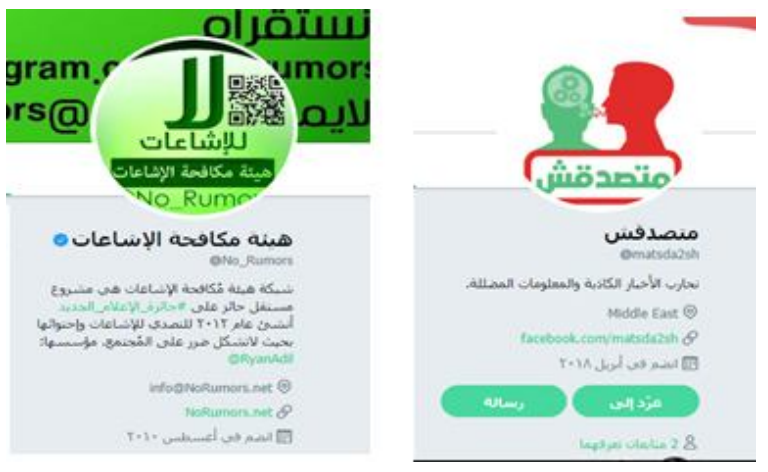

Figure 12: Accounts to Deny Rumors

The followed guidelines in order to consider the tweet content as a news are:

The news that seems to be a joke or the writer was not serious about it, are excluded.

- The tweets that is written as information not news, are excluded.

- $\quad$ The tweets that contain a question mark or ask for confirmation, are included.

Some of the news could not be labeled as credible or noncredible for some reasons:

- $\quad$ Did not find a trusted source to proof it or deny it.

- The news is spread in social media as an official news, but the official authority announces it after a few days. (For example, some accounts in twitter announced dismissal of Jorge Jesus the coach of AL-hilal club, after about three days of these rumors the club officially announced it).

- Half of the news content is credible but there is part of it that is non-credible, or the content tells half of the information and some parts are missing. For example, some users tweeted that New York State has approved to the " reproductive health act" law which allows abortion at any time from the Beginning of the pregnancy until the ninth month. Part of that news is true, but the law allows the abortion after the 24th week if $f$ the woman's life or health is in jeopardy.

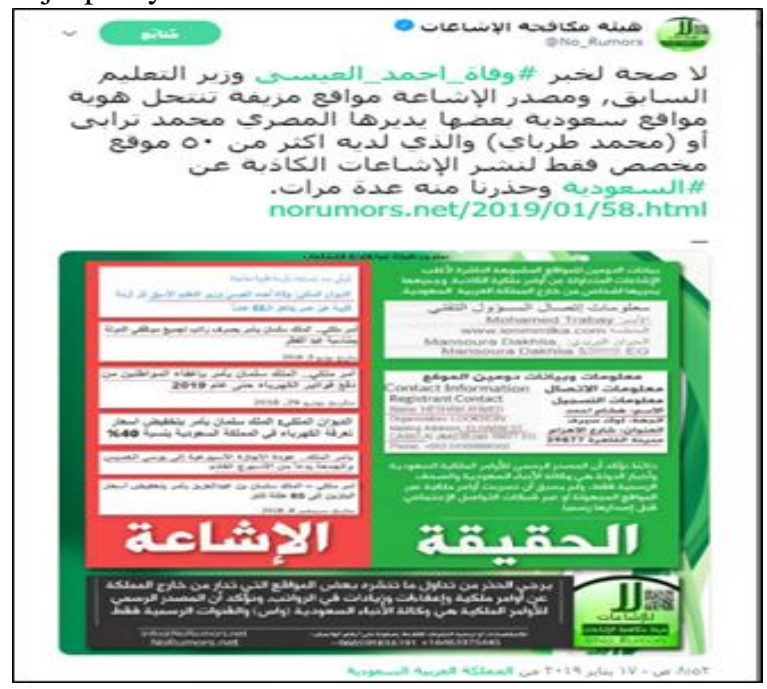

Figure 13: Proof the News Is Non-Credible

\section{FEATURES EXTRACTION}

This section will explain the features extraction mechanisms of the selected features in more details, showing the features that extracted from twitter's API followed as well as the features that need an extraction mechanisms which have been built to extract the features automatically instead of manually which will help to build a bigger data set and deal with it easily.

Twitter's API returns a set of data as json object called Meta Data such as: tweet text, postdate, user name...etc. Some features can get from this data directly and some other features are gotten indirect by perform simple calculations.

The set of features that are not identified in the meta data but can be derived from it indirectly, where the data attribute indicates to specific information but can derive another information or by perform some operations and calculations.

- Hashtags number in the tweet

- Tweet has URL

- Tweet has an image

- Number of question marks in tweet:

- Followers to followings ratio:

- Number of characters in tweet:

- Number of words in tweet

The set of features that are gotten directly by twitter's API:

- User has location

- User has description bio

- User has web page in bio

- User account is protected

- User followers' number

- User followings number

- User list number (The number of public lists that this user is a member of)

- User account created_at

- User's number of favorite tweets 
- User geo_enabled (user has enabled the possibility of geotagging their Tweets)

- User account is verified

- User's number of tweets

- User has default image?

- Is quoted tweet

- $\quad$ Number of retweets

- Number of favorites

Preprocessing methods have been applied to data to extract and compute some of the content features and before feeding to the classifier.

The main difficulty of performing and analyzing content of the Arabic social media is that the communication carried out used "spoken Arabic language" or "colloquial Arabic language" rather than the more formal Modern Standard Arabic (MSA), whereas the structure of the sentences and the vocabulary are very different and random than MSA. As has been mentioned before, the gathered data is for MSA only. Preprocessing steps include:

- Tokenization

Dividing the text into sequence of tokens as words.

- Non-Arabic removing

Removing any non-Arabic characters affecting the data and are not needed, such as English letters, numbers, symbols $\{\#, \$, ; \ldots$. etc. $\}$ by using our won code.

- $\quad$ Stop words removing

Stop words are the most common words in a language. Removing stop words save efforts for analyzing the content because they are useless and redundant, by building our won code and using existing Arabic stop word list from previous study (31).

- $\quad$ Stemming

Stemming process is reducing words to their root, such as "دراسة" which its root is "درس", is applied by using "AraNLP" java library that includes a sentence detector, tokenizer, light stemmer, root stemmer, part-of-speech tagger, word segmenter, normalizer, punctuation and diacritic remover (21).

The two types of stemming in the tools have been used Light stemmer and Root stemmer. Light stemming is done by truncating the prefixes and suffixes from the word and produce the stem, while the Root-based stemming is done by truncating prefixes and suffixes from the word and then matching with dictionary of root words to find the appropriate root (32).

Furthermore, the comments polarity has a high effect on credibility assessment.

Sentiment analysis also called Opinion Mining is a process of analyzing and understanding opinions about a given subject or product and classifying these opinions to (positive, negative or neutral). There are two categories of sentiment analysis: Machine Learning approach and Lexicon-Based approach. The approach that has been applied for Tweet's comments here is Lexicon-Based approach because the Machine Learning approach need to build a data set to train and test the classifier model and there is no existing Arabic dataset that can be used.

Each tweet's comment breaks up into words which take as tokens and after performing the preprocessing phase (section 6.2). Then by using "AraSenTi" a large-scale Twitter specific Arabic sentiment lexicon (33) has list of Arabic terms 116475 positive words and 108854 negative words with their sentiment score or weight. Also, the algorithm used to calculate the total polarity for each comment or tweet (34) as shown in.

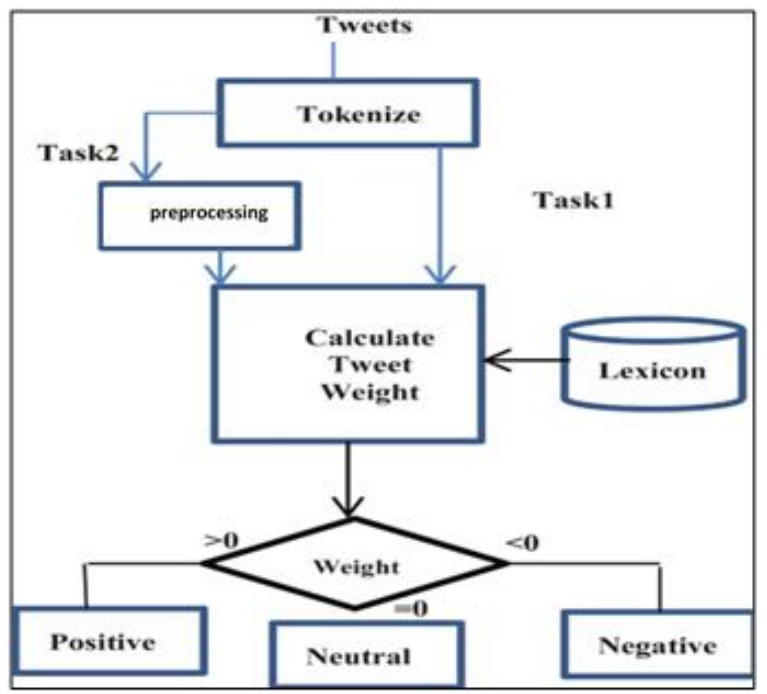

Figure 14: Architecture of Lexicon-Based Sentiment Analysis

The most comments are "spoken Arabic language" or "colloquial Arabic language" rather than the more formal Modern Standard Arabic (MSA) which we cannot control this case by collect only (MSA) comments as the tweets, but whereas the lexicon that has been used has a "colloquial Arabic" terms so that solve most of the case that does not stem correctly by stemmer.

The following features were pursued:

- Number of Negative/Positive Words in the Tweet: There are two different features indicate to number of positive and negative words that tweet has, which extract by using (33) lexicon that has been used in Tweet's comments polarity feature and our own code that compute number of positive and negative words in the tweet. Each tweet has two features of each negative and positive words number, one that has preprocessed by Light stemmer and the other by Root-based stemmer to compare the effect of each them.

- Tweet has Bad/Swear Words extract by using an existing Arabic bad word list (35) and our own code which checks that the tweet contains bad words. The feature value is 1 if tweet has bad word that indicates to True and 0 if does not that indicates to False.

- URL similarity: Most of previous related studies indicated to feature that if tweet has URL and few indicated to content or source of the linked URL, such as (8) who 
implement "Linking to authoritative or credible News" feature which the URL checked by human experts and assign it degree 1 to 4 , also (14) used Web of Trust (WOT) website that check links and providing crowdsourced reviews and rating of the source.

So, depending on that another method was implemented here to check the source content of the URL depends on calculating the similarity between the content of the tweet and the content of linked URL on the tweet called URL similarity feature. The strength of similarity between the tweet and the content of the URL indicates the news as more credible.

The feature extraction was done in two steps:

(i) Retrieving the content of the page through the link by using an existing code with some won modifications (36)which is retrieve the whole html source code of the page then by using won code that clear the file and get the content of the body only. Also was implemented the preprocessing methods that were mentioned in the aforementioned sections.

(ii) Similarity calculation which was implemented by using an existing code (37)(38) that implement the Jaccard Similarity. Jaccard similarity is a measures similarity and compares between sets. The Jaccard Similarity formula is the following:

$\mathrm{J}(\mathrm{A}, \mathrm{B})=(|\mathrm{A} \cap \mathrm{B}|) /(|\mathrm{A} \cup \mathrm{B}|)$

To calculate Jaccard similarity the page content document and tweet content must be processed with tokenization method for the document as a set of $\mathrm{N}$-grams in a vector space to be compared. $\mathrm{N}$-grams is sequence of $\mathrm{N}$ items of the given text document, the items can be adjacent characters or words. Three models of $\mathrm{N}$-grams implemented here two-character-grams, three-character-grams and twowords-grams so they have considered as three different features.

Some of the news tweets have images, sometimes these images are relevant to the news but sometimes they are not, in this section a mechanism was built to compare if the image content and the text of the tweet are in the same topic which can support the credibility of the news. To do so python packages was integrated which will be explained in the next points.

(i) The first step is to read the URL of the image from the tweet and fetch the image.

(ii) Image recognition:

To identify the objects or the content of an image ImageAI(39) was used, which is a Python package that help developers to build systems with some Computer Vision capabilities, such as image recognition, object detection, and video object tracking.

The package has 4 different models for image recognition:

- SqueezeNet: based on deep neural network developed by researchers at DeepScale, University of California, Berkeley, and Stanford University. (40).
The fastest among the four models but has moderate accuracy.

- ResNet50 based on Deep Residual Learning for Image Recognition developed by Microsoft Research (41) which won 1st place on the ILSVRC 2015 classification task \& the 1st places in ILSVRC\& COCO 2015 competitions on the tasks of ImageNet detection, ImageNet localization, $\mathrm{COCO}$ detection, and $\mathrm{COCO}$ segmentation.

Has both fast prediction time and good accuracy.

- InceptionV3: based on the Inception Architecture for computer vision developed by Google Brain team (42)which is an improvement for the InceptionV1 the winner of ILSVRC 2014.

Has slow prediction time but higher accuracy.

- DenseNet121: based on Convolutional Networks developed by Facebook AI Research (43), has slower prediction time but the highest accuracy among the four models.

To apply this model, the code from (44)was used with some modifications, with ResNet50 model which is the most suited model for this work. The prediction is a specified number of categories or objects that found in the image with the percentage probability for each prediction, in our case we chose 5 predictions. The predictions are in the English language, for that we used py-translator (45) free Google Translate API in python, to translate the output to the Arabic language.

The output of the previous step is list of words, in this step these words will be classified to different topics, also the tweet content will be classified and then compare if the tweet content and the image are in the same topic.

To do the classification step we used Arabic-News-ArticleClassification (46), Which is a classification model based on SVM with Stochastic Gradient Descent algorithm, classify the Arabic news to six different topics (religion, world, sport, society, politic, culture).

\section{CONDUCTED EXPERIMENTS}

This section will present the conducted experiments that implemented to select the classifier algorithm and features set that get the highest accuracy for the proposed system.

Different sets of features will be formed to use it in the classifier and test which set will give the highest accuracy, using all the features that were mentioned in the abovementioned sections

Feature Selection algorithms were used to eliminate irrelevant features that may affect the classifier algorithm in negative way and decrease its accuracy. The selection algorithm is divided into two parts the first is the Attribute Evaluator which evaluate each feature independently, the second part is the search method which find the best combination of features(47). 
Mai A. Fadel, International Journal of Advanced Trends in Computer Science and Engineering, 9(4), July - August 2020,6088 - 6102

In this work the following algorithms from WEKA GUI were used:

1. Best First \& Correlation-based Feature Selection in forward direction, selected 16 features.

2. Greedy Stepwise \& (Correlation-based Feature Selection) with no limitation to the number of features to be selected but with Threshold equals $1.7976931348623157 \mathrm{E} 308$, selected the same 16 features as the Best First.

3. Ranker \& Correlation Attribute Evaluation with no limitation to the number of features to be selected but with Threshold equals $-1.7976931348623157 \mathrm{E} 308$, ranked all the features and assigned each a weight within range 0 to 0.42344 .

4. Ranker \& Gain Ratio Attribute Evaluation with no limitation to the number of features to be selected but with Threshold equals $-1.7976931348623157 \mathrm{E} 308$, ranked all the features and assigned each a weight within range 0 to 0.1543404 .

5. Ranker \& Information Gain Attribute Evaluation with no limitation to the number of features to be selected but with Threshold equals

$-1.7976931348623157 \mathrm{E} 308$, ranked all the features and assigned each a weight within range 0 to 0.96985776 .

6. Ranker \& One Attribute Evaluation with no limitation to the number of features to be selected but with Threshold equals

$-1.7976931348623157 \mathrm{E} 308$ and with 10 folds, ranked all the features and assigned each a weight within range 53.8631 to 73.7307 .

7. Ranker \& Symmetrical Uncertainty Attribute Evaluation with no limitation to the number of features to be selected but with Threshold equals 1.7976931348623157E308, ranked all the features and assigned each a weight within range 0 to 0.1903566 .

8. Ranker \& ReliefF Attribute Evaluation with 10 neighbors, 1 seed, and Sigma equals 2, no limitation to the number of features to be selected but with Threshold equals $-1.7976931348623157 \mathrm{E} 308$, ranked all the features and assigned each a weight within range -0.000883 to 0.092936 .

By using Weka tool eight different classifier algorithms are used with 10 -fold cross validation with the sets of features in the experiments:

1) Decision Tree ID3 (J48)

2) Logistic Model Tree (LMT)

3) Random Tree (RT)

4) Bayes Networks (BNs)

5) Projective Adaptive Resonance Theory (PART)

6) Naïve Bayes (NB)

7) Decision Table

8) Random Forest (RF)

\section{RESULTS}

This section presents the experiments results to get the final model and features set for the purposed system. The experiments done with using each classifier algorithm with each feature set that was described previously .

Table 2: Experiments Results

\begin{tabular}{|r|l|l|l|}
\hline $\begin{array}{r}\text { Selection } \\
\text { algorithms }\end{array}$ & $\begin{array}{r}\text { Features } \\
\text { Num }\end{array}$ & Classifier & Accuracy \\
\hline $\begin{array}{r}\text { All the features are } \\
\text { included) }\end{array}$ & 36 & NB & $5.72 \%$ \\
\hline $\begin{array}{r}\text { Greedy Stepwise } \\
\text { \&Best First }\end{array}$ & 15 & MT & $4.17 \%$ \\
\hline Correlation & 8 & NB & $5.50 \%$ \\
\hline Gain Ratio & 10 & RT & $7.70 \%$ \\
\hline Info Gain & 13 & ART & $5.72 \%$ \\
\hline One & 10 & RT & $5.61 \%$ \\
\hline Attribute & & RT & $9.14 \%$ \\
\hline Rncertainty & 15 & ion Table & $1.32 \%$ \\
\hline
\end{tabular}

For these features selection algorithms that assign weight for each feature instead of selecting specific numbers of features (such as: Ranker \& CorrelationAttributeEval and \& Ranker $\&$ GainRatioAttributeEval) different number of top features from each set are used as a separated experiment $(8,10,13$ and 15 features) to try to get the highest accuracy with the minimum number of features.

Table 2 summarizes the results of the experiments by showing the highest accuracy that reached by each main set of features.

As shown in the abovementioned table, the highest accuracy reached is $81.35 \%$ using the top 8 feathers selected by Ranker \&ReliefF Attribute Eval selection algorithm and by the Decision Table classifier algorithm.

The features are the following::

1- $\quad$ User account is verified

2- User account created_at

3- Related image

4- $\quad$ Tweet has URL

5- $\quad$ Tweet has an image

6- $\quad$ User has web page in bio 
Mai A. Fadel, International Journal of Advanced Trends in Computer Science and Engineering, 9(4), July - August 2020,6088 - 6102

7- $\quad$ Hashtags number

8- Number of negative Words(lightStem)

This set manipulated by adding and deleting some features trying to reach higher accuracy. First, the selection algorithms almost gave the (related image) and (Tweet has an image) features the same weight and this might be because the actual effect in the (related image) feature is having an image not the topic of the image since it assign -1 to the tweets that don't include images and different numbers indicate if the image is in the same topic as the tweet to those include images, for that the (Tweet's Comments Polarity (lightStem)) which is the 9th top feature replaced the (related image) and that led to increase the accuracy to become $81.46 \%$. Second, when removing (User account created_at) feature the accuracy didn't change and this might be because it is considered as a nominal attribute and it is rarely to have more than one account created exactly at the same time, so it doesn't really have high effect.

After these two modifications the set ended up with 7 features which are:
1- User account is verified

2- $\quad$ Tweet has URL

3- Tweet has an image

4- User has web page in bio

5- Hashtags number

6- $\quad$ Number of negative Words(lightStem)

7- Tweet's Comments Polarity (lightStem)

The highest accuracy reached is $81.46 \%$. Comparing the proposed system to 3 previous studies on evaluating the credibility of Arabic news on Twitter. As shown in Table 3.

\section{CONCLUSION}

The paper presented a tool for evaluating Arabic news credibility by using classifiers. After conducting several experiments it was found that a system with 7 specific features running with the decision table classifier has the highest accuracy. Also the paper presented the creation of an initiation of Arabic data set for news credibility consisting of 906 records by automatically running feature extraction algorithms.

Table 3 :Comparison with existing studies

\begin{tabular}{|l|l|l|l|l|l|}
\hline & $\begin{array}{l}\text { Dataset } \\
\text { size }\end{array}$ & Accuracy & $\begin{array}{l}\text { Classifier } \\
\text { algorithm }\end{array}$ & $\begin{array}{l}\text { Number of } \\
\text { features }\end{array}$ & Feature selection algorithm \\
\hline$(7)$ & 199 tweets & $65.82 \%$ & ID3 Decision tree & 14 & Relief algorithm \\
\hline$(25)$ & $\begin{array}{l}9000 \\
\text { tweets }\end{array}$ & $\begin{array}{l}75.8 \\
\text { F-measure }\end{array}$ & Random Forest & 18 & Best First algorithm \\
\hline$(10)$ & 800 tweets & $90.81 \%$ & ID3 Decision tree & 13 & None \\
\hline Proposed System & 906 tweets & $81.46 \%$ & Decision table & 7 & ReliefF algorithm \\
\hline
\end{tabular}

Future work includes the following: (i) Adding the similarity with trusted source feature which on some other research have a high effect and increase the accuracy of the model such (10)(8)(16). It will need more effort to build it because in this work the tweets dataset are in different topics like sport, economy, health ..etc. and some of the news like the political ones should be proved by trusted source in specific country, so there will be need to specify a large list of trusted source to each on of this topic and from each country, (ii) Analyzing the content of the images in the tweets in a better way with more details not only the topic they belong to, (iii) Increasing the size of the dataset to train the model better, (iv) Building a website version of the tool to make it easier to access it by the users.

\section{ACKNOWLEDGMENT}

I wish to acknowledge the excellent technical work done by the Bachelor holders SAHAR SALEM BAJANDOUH and AREEJ SALEH ALZHRANI during their CS senior project.

\section{REFERENCES}

[1] Internet World Stats, 2018, available at https://www.census.gov/.
[2]International Telecommunication Union . ITU, 2015, available at https://www.itu.int/en/Pages/default.aspx.

[3]Saudi CITC. Saudi Communications and Information Technology Commission website, 2018, availabel at http://www.citc.gov.sa/ar/mediacenter/pressreleases/Pages/2 017062001.aspx.

[4] Abdullah, Rasha, et al., The Credibility of Newspapers, Television News, And Online News, published by Journalism and Mass Communications Conference. 9, 2015.

[5] Boyd, Danah and Ellison, Nicole, Social Network Sites: Definition, History, and Scholarship, 2007.

- [6] Kang, Byungkyu, O’Donovan, John and Hollerer, Tobias . Modeling Topic Specific Credibility in Twitter,published by Association for Computing Machinery,Lisbon, Portugal : s.n., 2012.

[7]Almansour, Amal. Credibility Assessment For Arabic Micro-Blogs Using Noisy Labels, London : s.n., 2016.

[8] Al-Eid, Rasha, Al-Khalif, Hend and Al-Salman, AbdulMalik. Measuring The Credibility of Arabic Text Content in Twitter, Riyadh,Saudi Arabia : IEEE, 2010.

[9] Zhao, Zhe , Resnick, Paul and Mei, Qiaozhu, Enquiring Minds: Early Detection of Rumors in Social, Florence, Italy : s.n., 2015.

[10] Sabbeh, Sahar and Baatwah, Sumaia. "ARABIC NEWS CREDIBILITY ON TWITTER: AN ENHANCED MODEL 
Mai A. Fadel, International Journal of Advanced Trends in Computer Science and Engineering, 9(4), July - August 2020,6088 - 6102

USING HYBRID FEATURES", Journal of Theoretical and Applied Information Technology, 2018.

[11]Xia, Xin, et al., Information Credibility on Twitter, 2012.

[12]Counts, Scott, et al., Tweeting is Believing? Understanding Microblog, Seattle, Washington, USA : CSCW, 2012.

[13] Radev , Dragomir , et al., Rumor has it: Identifying Misinformation in Microblogs, Edinburgh, Scotland, UK : s.n., 2011.

[14]Gupta, Aditi, et al., TweetCred: Real-Time Credibility Assessment of Content on Twitter ,2015.

[15] Fairbanks, James , et al., Credibility Assessment in the News: Do we need to read? Marina Del Rey, CA, USA : s.n., 2018.

[16] Lorek, Krzysztof, et al., AUTOMATED CREDIBILITY ASSESSMENT ON TWITTER, 2015.

[17] Juffinger, Andreas, Granitzer, Michael and Lex, Elisabeth. Blog Credibility Ranking by Exploiting Verified Content. Madrid, Spain : s.n., 2009.

[18]Ray, Sunil,analytics vidhya,2017, available at https://www.analyticsvidhya.com/blog.

[19] Pawlak, Zdzisław. Rough sets, decision algorithms and Bayes' theorem, s.l. : Elsevier, 2002.

[20]Ahamdi, R, et al., A study on part of speech tagging, 2011.

[21] Althobaiti, Maha, Kruschwitz, Udo and Poesio, Massimo, AraNLP,Colchester, UK : s.n., 2014.

[22] Stoltzfus, Justin. techopedia,available at https://www.techopedia.com/why-is-data-annotation-

important-in-some-machine-learning-projects/7/33331.

[23] Castillo, Carlos, Mendoza, Marcelo and Poblete, Barbara,Information Credibility on Twitter,Hyderabad, India : s.n., 2011.

[24]Gupta, Aditi, TweetCred, 2015.

[25] El Ballouli , Rim, et al., CAT: Credibility Analysis of Arabic Content on Twitter, 2017.

[26] Elrashidy, Mohammed, et al., SENTIMENT ANALYSIS FOR ARABIC AND ENGLISH DATASETS, 2015.

[27] Hardalov, Momchil , Koychev, Ivan and Nako,Preslav, In Search of Credible News, 2017.

[28]MK Lab,image-verification-corpus. github, 2015, available at https://github.com/MKLab-ITI/imageverification-corpus.

[29]Xu , Wei and Tabassum, Jeniya, Twitter API tutorial, 2018, available at

http://socialmedia-class.org/twittertutorial.html.

[30]Ravindran, Rohith, stackoverflow, 2018, available at https://stackoverflow.com/questions/29928638/gettingtweet-replies-to-a-particular-tweet-from-a-particular-user. [31]Abu El-Khair, Ibrahim,Effects of Stop Words Elimination for AIR: A Comparative Study, Egypt : s.n., 2017.

[32] Abuata, Belal Mustafa and Sembok, Tengku,Arabic Word Stemming Algorithms and Retrieval Effectiveness, London : WCE 2013, 2013.

[33] AlTwairesh , Nora, AlKhalifa, Hend and AlSalman, AbdulMalik,AraSenti Lexicon, Riyadh : s.n., 2016.
[34]Abdulla, Nawaf, et al.,Towards Improving the Lexicon-Based Approach for Arabic Sentiment Analysis, s.l. : IGI, 2014.

[35] data.world, Dirty Naughty Obscene and Otherwise Bad Words in Arabic, 2017, available at

https://data.world/wordlists/dirty-naughty-obscene-andotherwise-bad-words-in-

arabic/workspace/file?filename=Arabic.csv.

[36] mkyong, How to get URL content in Java, 2012, available at https://www.mkyong.com/java/how-to-get-urlcontent-in-java/.

[37] Sunkaranen, Tarun, Document Similarity. Jupyter notebook, available at

https://nbviewer.jupyter.org/github/TarunSunkaraneni/Docu ment-Similarity/blob/master/Document\%20Similarity.ipynb.

[38]Sunkaraneni, Tarun, Document Similarity. github, 2018, available at https://github.com/TarunSunkaraneni/ Document-Similarity.

[39]Deep Quest AI, ImageAI, available at

http://www.imageai.org/.

[40] N. Iandola, Forrest, et al., SQUEEZENET: ALEXNET-LEVEL ACCURACY WITH,2016.

[41]He, Kaiming , et al., Deep Residual Learning for Image Recognition, 2015.

[42] Szegedy, Christian, et al., Rethinking the Inception Architecture for Computer Vision, 2016.

[43] Huang, Gao, Liu, Zhuang and Laurens van der Maaten,Densely Connected Convolutional Networks, 2018.

[44] Olafenwa, Moses,Image Recognition with 10 lines of code.medium, 2018, available at

https://medium.com/@guymodscientist/image-prediction-

with-10-lines-of-code-3266f4039c7a.

[45] py-translator 2.1.9,py-translator 2.1.9,2019, available at https://pypi.org/project/py-translator/.

[46]Ziani, Saïd,Arabic News Article Classification. GitHub, 2018, available at https://github.com/saidziani/Arabic-News-Article-

Classification.

[47]S. Dinakaran, Dr. P. Ranjit Jeba Thangaiah, Role of Attribute Selection in Classification,2013. 